\title{
$S$ parameter and parity doubling below the conformal window
}

\section{David Schaich* for the Lattice Strong Dynamics (LSD) Collaboration ${ }^{\dagger}$}

Physics Department \& Center for Computational Science, Boston University, Boston, MA 02215

Department of Physics, University of Colorado, Boulder, CO $80309^{*}$

Email: schaich@pizero.colorado.edu

\begin{abstract}
Recently the Lattice Strong Dynamics Collaboration reported a reduction of the electroweak $S$ parameter for SU(3) gauge theory with $N_{f}=6$ fermions in the fundamental representation, compared to scaled-up QCD. Here I provide additional details of our calculation. I discuss our use of conserved lattice currents; the relation to vector-axial parity doubling; finite-volume effects; and the sensitivity of our results to the number of fermion doublets with chiral electroweak couplings. Results presented here include additional data, and do not affect our previously-published conclusions.
\end{abstract}

XXIX International Symposium on Lattice Field Theory

10-16 July 2011

Squaw Valley, Lake Tahoe, California

\footnotetext{
* Speaker.

†http://www.yale.edu/LSD

†Present address
} 


\section{Introduction}

The application of lattice gauge theory to strongly-interacting physics beyond QCD is at present a very active field [1]. While much of the current interest is motivated by the possibility that new strong dynamics may play a role in electroweak symmetry breaking [2,3], improving our general understanding of strong dynamics is an important theoretical goal in its own right.

The standard picture of strongly-interacting $\mathrm{SU}(N)$ gauge theories is that as we increase the number $N_{f}$ of fermions in a given representation, an infrared fixed point will develop at some critical $N_{f}^{(c)}$. For $N_{f} \geq N_{f}^{(c)}$ (up to the loss of asymptotic freedom) the system is IR-conformal. Approximately-conformal systems with $N_{f} \lesssim N_{f}^{(c)}$ may possess the dynamical scale separation that characterizes "walking" theories, as well as parity doubling between vector $(V)$ and axial-vector (A) spectra that can reduce the electroweak $S$ parameter to phenomenologically viable values [4].

The Lattice Strong Dynamics Collaboration approaches these questions by using QCD as a baseline. We consider SU(3) gauge theory and steadily increase the number of fundamental fermions, comparing our results against the familiar case $N_{f}=2$. We use computationally expensive domain wall fermions for better control over lattice artifacts. Our first studies focused on the $N_{f}=6$ model, which while not truly walking exhibits some of the associated phenomena: by matching IR scales between $N_{f}=2$ and $N_{f}=6$ calculations, we observed an enhancement in the $N_{f}=6$ chiral condensate [5] and a reduction of the $S$ parameter relative to scaled-up QCD [6]. Here I provide additional details of our $S$ parameter calculation that were not discussed in Ref. [6]. Results presented here also include additional data, and do not affect the conclusions of Ref. [6].

We can identify three main ingredients in our expression for the $S$ parameter,

$$
S=4 \pi N_{D} \lim _{Q^{2} \rightarrow 0} \frac{d}{d Q^{2}} \Pi_{V-A}\left(Q^{2}\right)-\Delta S_{S M}
$$

The term $\Delta S_{S M}$ accounts for the three Nambu-Goldstone bosons (NGBs) eaten by the $W^{ \pm}$and $Z$, and is discussed in detail by Ref. [6]. In Section 2 I review our calculation of the transverse $V-A$ polarization function $\Pi_{V-A}\left(Q^{2}\right)$, and relate it to the vector and axial spectra in Section 3. Finally, $N_{D}$ is the number of doublets with chiral electroweak couplings; in Section 4 I show how it affects our results for the $S$ parameter.

\section{Currents and correlators}

On the lattice, the transverse $V-A$ polarization function $\Pi_{V-A}\left(Q^{2}\right)$ is determined from

$$
\begin{aligned}
\Pi_{V-A}^{\mu \nu}(Q) & =\left(\delta^{\mu v}-\frac{\widehat{Q}^{\mu} \widehat{Q}^{v}}{\widehat{Q}^{2}}\right) \Pi_{V-A}\left(Q^{2}\right)-\frac{\widehat{Q}^{\mu} \widehat{Q}^{v}}{\widehat{Q}^{2}} \Pi_{V-A}^{L}\left(Q^{2}\right) \\
& =Z \sum_{x} e^{i Q \cdot(x+\widehat{\mu} / 2)} \operatorname{Tr}\left[\left\langle\mathscr{V}^{\mu a}(x) V^{v b}(0)\right\rangle-\left\langle\mathscr{A}^{\mu a}(x) A^{v b}(0)\right\rangle\right] .
\end{aligned}
$$

Here $\widehat{Q}=2 \sin (\pi n / L)$ are lattice momenta, while $Q=2 \pi n / L$; these are spacelike $Q^{2}=-q^{2}>0$. The current correlators mix two types of domain wall currents. $V^{\mu a}$ and $A^{\mu a}$ are non-conserved 
"local" currents defined on the domain walls; in terms of five-dimensional fermion fields $\Psi(x, s)$,

$$
\begin{aligned}
& V^{\mu a}(x)=\frac{1}{2}\left\{\bar{\Psi}\left(x, L_{s}-1\right) \gamma^{\mu}\left(1+\gamma^{5}\right) \tau^{a} \Psi\left(x, L_{s}-1\right)+\bar{\Psi}(x, 0) \gamma^{\mu}\left(1-\gamma^{5}\right) \tau^{a} \Psi(x, 0)\right\} \\
& A^{\mu a}(x)=\frac{1}{2}\left\{\bar{\Psi}\left(x, L_{s}-1\right) \gamma^{\mu}\left(1+\gamma^{5}\right) \tau^{a} \Psi\left(x, L_{s}-1\right)-\bar{\Psi}(x, 0) \gamma^{\mu}\left(1-\gamma^{5}\right) \tau^{a} \Psi(x, 0)\right\} .
\end{aligned}
$$

The conserved currents $\mathscr{V}^{\mu a}$ and $\mathscr{A}^{\mu a}$ are point-split, and summed over the fifth dimension:

$$
\begin{gathered}
\mathscr{V}^{\mu a}(x)=\sum_{s=0}^{L_{s}-1} j^{\mu a}(x, s) \quad \mathscr{A}^{\mu a}(x)=\sum_{s=0}^{L_{s}-1} \operatorname{sign}\left(s-\frac{L_{s}-1}{2}\right) j^{\mu a}(x, s), \\
j^{\mu a}(x, s)=\frac{1}{2}\left\{\bar{\Psi}(x+\widehat{\mu}, s)\left(1+\gamma^{\mu}\right) U_{x, \mu}^{\dagger} \tau^{a} \Psi(x, s)-\bar{\Psi}(x, s)\left(1-\gamma^{\mu}\right) U_{x, \mu} \tau^{a} \Psi(x+\widehat{\mu}, s)\right\} .
\end{gathered}
$$

The Fourier transform in Eqn. 2.1 involves $(x+\widehat{\mu} / 2)$ because the conserved currents are point-split on the link $(x, x+\widehat{\mu})$. The flavor matrices $\tau^{a}$ are normalized to $\operatorname{Tr}\left[\tau^{a} \tau^{b}\right]=\delta^{a b} / 2$.

Although the conserved and local currents must agree in the continuum limit, at finite lattice spacing only the former satisfy a Ward identity $\left(\widehat{Q}_{\mu} \Pi_{V V}^{\mu v}=0\right.$, Fig. 1). Because the correlators involve both currents, Eqn. 2.1 includes the renormalization factor $Z$, which we compute nonperturbatively, $Z=0.85(0.73)$ for $N_{f}=2$ (6). Our chiral lattice fermions ensure that $Z=Z_{A}=Z_{V}$.
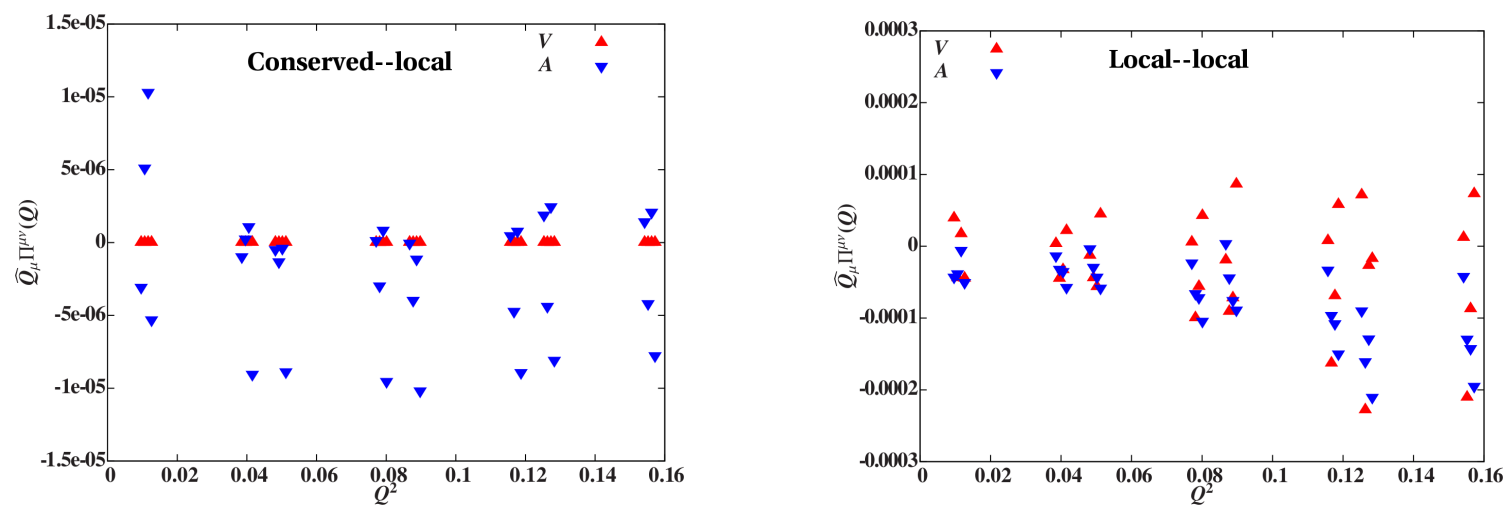

Figure 1: On every configuration, $\widehat{Q}_{\mu} \Pi_{V V}^{\mu v}=0$ when one conserved current is used in each correlator (left), but not when only non-conserved local currents are used (right). The horizontal offsets around each $Q^{2}$ value distinguish different $v$.

In principle, it would be best to work entirely with the conserved currents $\mathscr{V}^{\mu a}$ and $\mathscr{A}^{\mu a}$ instead of using the mixed correlators in Eqn. 2.1. In practice, evaluating conserved-conserved correlators such as $\left\langle\mathscr{V}^{\mu a}(x)^{v b}(0)\right\rangle$ requires $\mathscr{O}\left(L_{s}\right)$ inversions, increasing the computational cost of the calculation by roughly an order of magnitude. As emphasized in Ref. [7], lattice artifacts cancel in the $V-A$ difference of the mixed correlators, allowing us to use these less expensive quantities. This is illustrated in the left panel of Fig. 2: even though $\Pi^{\mu v} \widehat{Q}_{v} \neq 0$ since $V^{v a}$ and $A^{v a}$ are not conserved, $\left[\Pi_{V V}^{\mu v}\left(Q^{2}\right)-\Pi_{A A}^{\mu v}\left(Q^{2}\right)\right] \widehat{Q}_{v} \approx 0$. In the right panel, we see that this does not hold if we use only local currents in the correlators. 

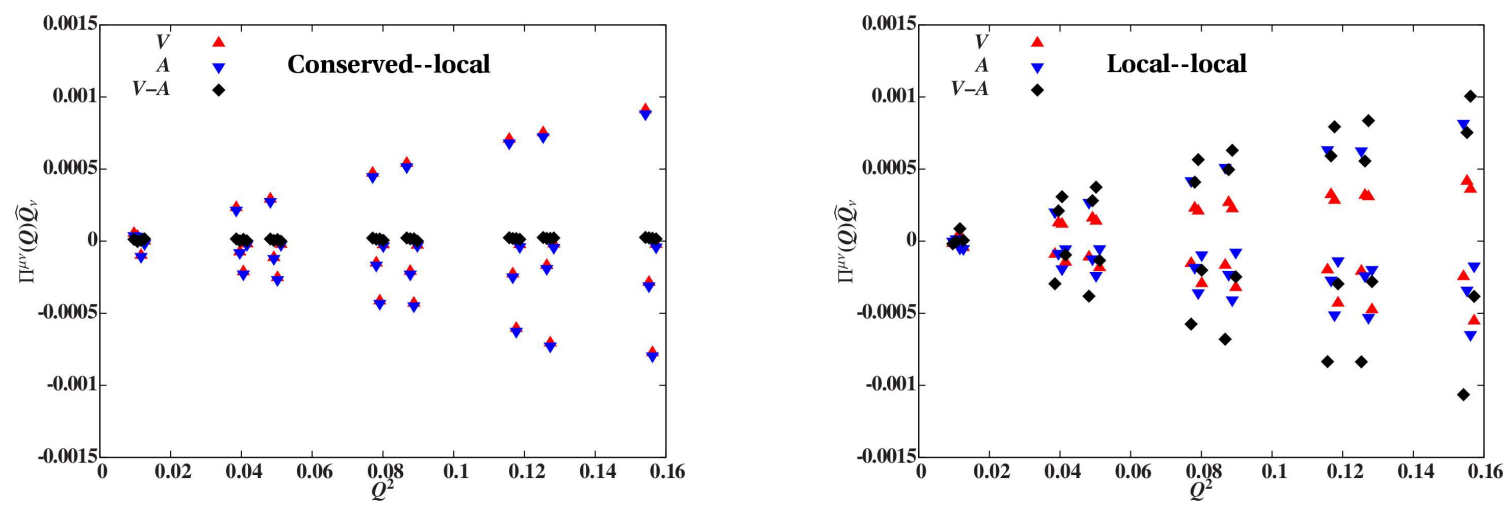

Figure 2: On every configuration, lattice artifacts $\Pi^{\mu v} \widehat{Q}_{v} \neq 0$ cancel in the $V-A$ difference when one conserved current is used in each correlator (left), but not when only non-conserved local currents are used (right). The horizontal offsets around each $Q^{2}$ value distinguish different $\mu$.

\section{Parity doubling and finite volume effects}

Because chiral perturbation theory cannot reliably be applied to our $N_{f}=6$ calculations [8], we extract the slope $\Pi_{V-A}^{\prime}(0)$ by fitting our data to a simple four-parameter rational function,

$$
\Pi_{V-A}\left(Q^{2}\right)=\frac{a_{0}+a_{1} Q^{2}}{1+b_{1} Q^{2}+b_{2} Q^{4}} .
$$

This "Padé(1,2)" functional form has the correct asymptotic behavior $\Pi_{V-A}\left(Q^{2}\right) \sim Q^{-2}$ at large $Q^{2}$, and also resembles the single-pole dominance approximation to the $V-A$ dispersion relation

$$
\Pi_{V-A}\left(Q^{2}\right)=-F_{P}^{2}+\frac{Q^{2}}{12 \pi} \int_{0}^{\infty} \frac{d s}{\pi}\left[\frac{R_{V}(s)-R_{A}(s)}{s+Q^{2}}\right] .
$$

( $F_{P}$ is the pseudoscalar decay constant.) That is, with the single-pole dominance approximation $R(s)=12 \pi^{2} F^{2} \delta\left(s-M^{2}\right)$, this dispersion relation becomes

$$
\Pi_{V-A}^{(\text {pole })}\left(Q^{2}\right)=-F_{P}^{2}+\frac{Q^{2} F_{V}^{2}}{M_{V}^{2}+Q^{2}}-\frac{Q^{2} F_{A}^{2}}{M_{A}^{2}+Q^{2}},
$$

which reproduces the form of Eqn. 3.1 when we apply the corresponding approximation to the first Weinberg sum rule, $F_{P}^{2}=F_{V}^{2}-F_{A}^{2}$. Because the lattice data contain information about the entire spectrum, the fit parameters in Eqn. 3.1 do not directly correspond to the combinations of meson masses and decay constants predicted by the pole-dominance Eqn. 3.3.

Uncorrelated fits of our data to Eqn. 3.1 produce stable results with $\chi^{2} /$ dof $\ll 1$ as we vary the $Q^{2}$ fit range. Our results for $\Pi_{V-A}^{\prime}(0)$ are shown as colored points in the left panel of Fig. 3. The black points in that plot are pole-dominance predictions based on Eqn. 3.3. Both the direct fit results and the pole-dominance predictions show a reduction for $N_{f}=6$ compared to $N_{f}=2$ at light pseudoscalar masses $M_{P} \lesssim M_{V 0}$, where $M_{V 0}$ is the vector meson mass in the chiral limit. The pole-dominance predictions are systematically lower than the direct results, consistent with the expectation that states neglected by the single-pole dominance approximation would provide additional positive contributions. 

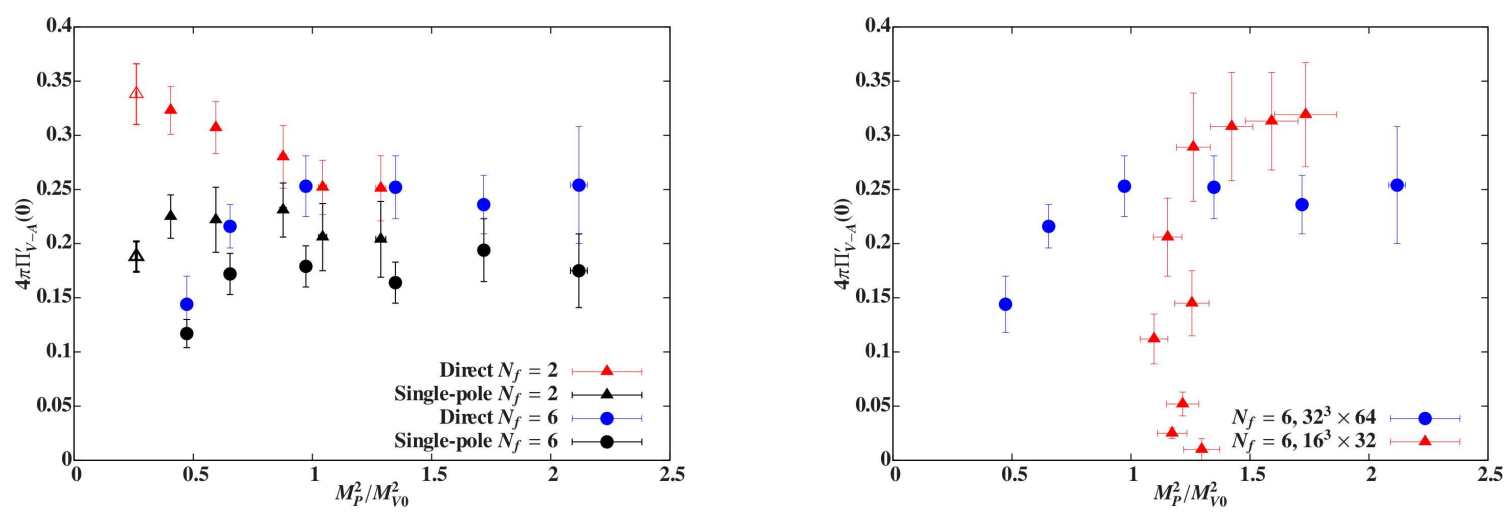

Figure 3: The slope of $\Pi_{V-A}\left(Q^{2}\right)$ at $Q^{2}=0$, plotted versus $M_{P}^{2} / M_{V 0}^{2}$. Left: $N_{f}=2$ and 6 results on $32^{3} \times 64$ volumes from direct fits to Eqn. 3.1 (colored), compared to pole-dominance predictions (black). Right: $N_{f}=6$ results on $16^{3} \times 32$ and $32^{3} \times 64$ volumes.

The lightest $N_{f}=2$ points in Fig. 3 are empty because they correspond to a fermion mass $m$ so small that finite-volume effects may be significant. Finite-volume effects are a concern for the $S$ parameter calculation because they can produce spurious parity doubling that artificially reduces $\Pi_{V-A}^{\prime}(0)$. This is illustrated in the right panel of Fig. 3 for $N_{f}=6$ calculations on $16^{3} \times 32$ volumes: $\Pi_{V-A}^{\prime}(0) \rightarrow 0$ as $m \rightarrow 0$, which would naïvely suggest a negative $S$ parameter from Eqn. 1.1. The associated distortion of the spectrum provides clear evidence that this is merely a finite-volume effect: as $m$ decreases, the $16^{3} \times 32$ pseudoscalar mass $M_{P}$ freezes around $M_{P}^{2} \approx 1.2 M_{V 0}^{2}$, which is not the case for the $32^{3} \times 64$ results also shown in the plot.

Returning to the lightest $N_{f}=2$ points, the pole-dominance prediction for $\Pi_{V-A}^{\prime}(0)$ decreases due to spurious parity doubling from finite-volume effects. However, we do not see a similar reduction in the direct fit result. Instead, this point clearly continues the trend established at heavier masses, and the corresponding $N_{f}=2$ results for $S$ (Fig. 4, below) reproduce the prediction obtained by scaling up QCD phenomenology, $\lim _{M_{P}^{2} \rightarrow 0} S=0.32(3)$ [4]. This suggests that the Padé fits may be less sensitive than spectral quantities to these finite-volume effects, increasing our confidence that the reduction observed for $N_{f}=6$ is physical.

\section{4. $S$ parameter results}

Realistic models of dynamical electroweak symmetry breaking must produce exactly three massless NGBs to be eaten by the $W^{ \pm}$and $Z$. Any additional pseudo-Nambu-Goldstone bosons (PNGBs) must acquire masses from standard-model and other (e.g., extended-technicolor) interactions in order to satisfy experimental constraints. On the lattice, however, we perform calculations with $N_{f}^{2}-1$ degenerate massive PNGBs. When we use Eqn. 1.1 to determine the $S$ parameter from the $\Pi_{V-A}^{\prime}(0)$ results shown in Fig. 3, the $\Delta S_{S M}$ term removes the contribution only of the three would-be NGBs. (To be more precise, the $I_{3}=0$ NGB does not contribute, and $\Delta S_{S M}$ cancels the contribution of the $\left|I_{3}\right|=1$ pair.) The remaining $N_{f}^{2}-4$ PNGBs introduce chiral-log terms $\propto \log \left[M_{V 0}^{2} / M_{P}^{2}\right]$ that would diverge in the chiral limit $M_{P}^{2} \rightarrow 0$.

Fig. 4 presents our $S$ parameter results for $N_{f}=2$ and 6, considering two possible values of $N_{D}$ for $N_{f}=6$. The plot on the left presents the case in which every fermion possesses chiral 
electroweak couplings, $N_{D}=N_{f} / 2=3$. The minimal case in which only a single doublet has chiral couplings $\left(N_{D}=1\right)$ is shown on the right. In both cases the $N_{f}=6$ results show a reduction compared to rescaling $N_{f}=2$, before diverging in the chiral limit. With $N_{D}=1$ the $S$ parameter can be significantly closer to the experimental value $S \approx-0.15(10)$ for $M_{H}^{(r e f)} \sim 1 \mathrm{TeV}$ [9].
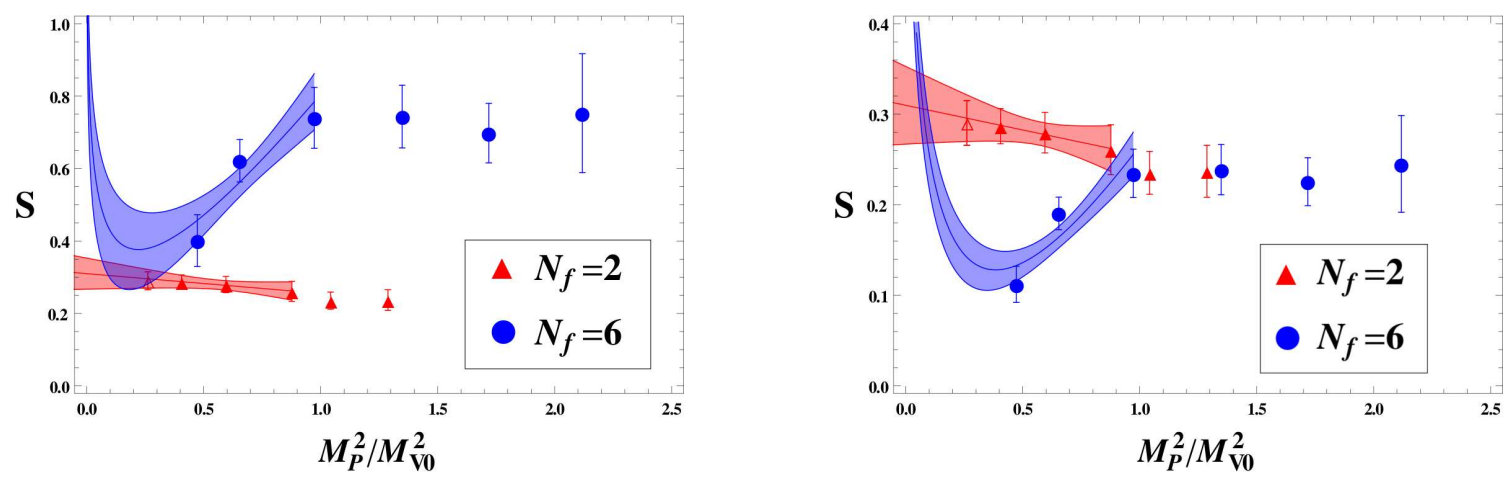

Figure 4: $S$ parameter for $N_{f}=2$ and 6, for the maximum $N_{D}=3$ (left) and minimum $N_{D}=1$ (right). The bands correspond to fits explained in the text.

To guide the eye, we include in Fig. 4 simple linear fits accounting for the $N_{D}$-dependent chiral-log divergence that remains for $N_{f}>2$. We fit the lightest three solid points to the form

$$
S=A+B x+\frac{\sharp-1}{12 \pi} \log (1 / x)
$$

where $x \equiv M_{P}^{2} / M_{V 0}^{2}$ and $\sharp$ counts the pairs of PNGBs with $I_{3} \neq 0$,

$$
\sharp=\left(\frac{N_{f}}{2}\right)^{2} \quad \text { for } N_{D}=N_{f} / 2 \quad \sharp=2 N_{f}-3 \quad \text { for } N_{D}=1 .
$$

The blue $N_{f}=6$ curves allow us to estimate the fermion mass $m$ at which we could directly observe chiral log effects. The necessary $m$ is too small for us to explore on our present $32^{3} \times 64$ volumes.

Again, in a realistic phenomenological context, we must have only three massless NGBs, with $N_{f}^{2}-4$ massive PNGBs. To estimate a definite value for the $N_{f}=6 S$ parameter in this situation, we can imagine freezing the masses of all $N_{f}^{2}-4$ PNGBs at some finite value (such as $M_{P}^{2}=0.38 M_{V 0}^{2}$ at the minimum of the $N_{D}=1$ blue curve in Fig. 4), and then taking only the three NGBs to the chiral limit $M_{P}^{2} \rightarrow 0$. A qualitative picture of this scenario is sketched in Fig. 5 .

\section{Acknowledgments}

I thank the members of the LSD Collaboration for many useful discussions, and review of this contribution: T. Appelquist, R. Babich, R. Brower, M. Buchoff, M. Cheng, M. Clark, S. Cohen, G. Fleming, J. Kiskis, M. Lin, H. Na, E. Neil, J. Osborn, C. Rebbi, S. Syritsyn, P. Vranas, G. Voronov, J. Wasem and O. Witzel. This work was supported by the U.S. Department of Energy (DOE) through grants DE-FG02-91ER40676 and DE-FG02-04ER41290; the Lawrence Livermore National Laboratory Institutional Computing Grand Challenge program; the DOE Scientific Discovery through Advanced Computing program through the USQCD Collaboration; ${ }^{1}$ the

\footnotetext{
${ }^{1}$ http: / / www . usqcd.org
} 


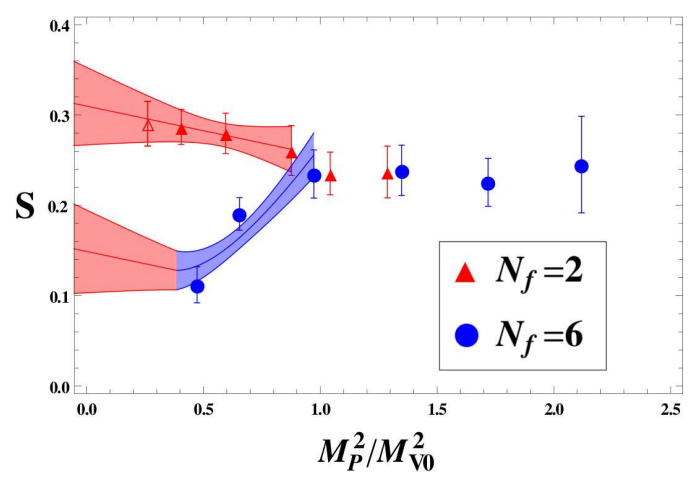

Figure 5: $S$ parameter for $N_{f}=2$ and 6 with $N_{D}=1$, imagining that we freeze the masses of all $N_{f}^{2}-4$ PNGBs at $M_{P}^{2}=0.38 M_{V 0}^{2}$, as described in the text.

U.S. National Science Foundation through TeraGrid resources provided by the National Institute for Computational Sciences under grant number TG-MCA08X008; ${ }^{2}$ and Boston University's Scientific Computing Facilities.

\section{References}

[1] L. Del Debbio, “The conformal window on the lattice”, PoS Lattice 2010 (2011) 004, arXiv:1102.4066 [hep-lat].

[2] C. T. Hill and E. H. Simmons, "Strong dynamics and electroweak symmetry breaking", Phys. Rept. 381 (2003) 235-402, arXiv: hep-ph/0203079 [hep-ph].

[3] S. Rychkov, "EWSB Theory on the Eve of Higgs Boson Exclusion/Discovery", PoS EPS-HEP2011 (2011) 029, arXiv:1109.1180 [hep-ph] .

[4] M. E. Peskin and T. Takeuchi, "Estimation of oblique electroweak corrections", Phys. Rev. D46 (1992) 381-409.

[5] LSD Collaboration, T. Appelquist, A. Avakian, R. Babich, R. C. Brower, M. Cheng, M. A. Clark, S. D. Cohen, G. T. Fleming, J. Kiskis, E. T. Neil, J. C. Osborn, C. Rebbi, D. Schaich, and P. Vranas, “Toward TeV Conformality", Phys. Rev. Lett. 104 (2010) 071601, arXiv:0910.2224 [hep-ph] .

[6] LSD Collaboration, T. Appelquist, R. Babich, R. C. Brower, M. Cheng, M. A. Clark, S. D. Cohen, G. T. Fleming, J. Kiskis, M. Lin, E. T. Neil, J. C. Osborn, C. Rebbi, D. Schaich, and P. Vranas, "Parity Doubling and the S Parameter Below the Conformal Window", Phys. Rev. Lett. 106 (2011) 231601, arXiv:1009.5967 [hep-ph].

[7] RBC and UKQCD Collaboration, P. A. Boyle, L. Del Debbio, J. Wennekers, and J. M. Zanotti, "The S Parameter in QCD from Domain Wall Fermions", Phys. Rev. D81 (2010) 014504, arXiv:0909.4931 [hep-lat].

[8] LSD Collaboration, E. T. Neil, A. Avakian, R. Babich, R. C. Brower, M. Cheng, M. A. Clark, S. D. Cohen, G. T. Fleming, J. Kiskis, J. C. Osborn, C. Rebbi, D. Schaich, and P. Vranas, "Lattice study of ChPT beyond QCD”, PoS CD09 (2009) 088, arXiv:1002.3777 [hep-lat ] .

[9] K. Nakamura et al., "Review of particle physics", J. Phys. G37 (2010) 075021.

\footnotetext{
${ }^{2}$ http: //www.xsede.org
} 